\title{
Conrad Geßners Beziehungen zu einem kroatischen Gelehrten
}

\author{
Von Dr. Lavoslav Glesinger, Zagreb (Jugoslawien)
}

Die bedeutungsvolle Rolle Conrad Gessners im Geistesleben seiner Zeit kommt im Briefwechsel mit seinen Zeitgenossen ${ }^{1}$ deutlich zum Ausdruck. Die Briefe Gessners, von denen Haller anerkennend sagt: «... valde tamen placent varietate, candore et eruditione, quibus undique plenae sint» ${ }^{2}$, sind eine Fundgrube für die Erforschung nicht nur der Geschichte der Medizin und Naturwissenschaften im 16. Jahrhundert, sondern des gesamten Geisteslebens jener Zeit. Mit Recht weist Sudhoff auf den Wert dieses «fast unvergleichlich wichtigen Briefwechsels» hin ${ }^{3}$, dessen Erforschung ungemein aufschlußreiche Ergebnisse zutage gefördert hat. Die Anzahl der Männer, mit denen Gessner Beziehungen unterhielt, ist sehr ansehnlich : wir finden darunter - entsprechend dem vielseitigen Interessenkreis Gessners - Vertreter der verschiedensten. Wissenschaften aus aller Herren Ländern. Und doch umfaßt diese Sammlung nicht den gesamten Briefwechsel Gessners. Eine Anzahl seiner Schriftstücke (und sie wird wohl nicht unbedeutend sein) hat in dieser Sammlung keine Aufnahme gefunden und ist zum Teil bisher überhaupt nicht bekannt geworden ${ }^{4}$. Andere Briefe wurden vielleicht einmal veröffentlicht und liegen in irgendeinem alten und heute kaum auffindbaren Druckwerk verborgen. Diese Briefe bleiben wegen ihrer Unzulänglichkeit der gegenwärtigen Forschung meist unbekannt. $\mathrm{Da} \beta$ es darunter auch solche Briefe gibt, die Nachrichten über bisher unbekannte Beziehungen Gessners enthalten oder sonstwie für die Forschung besonders aufschlußreich sind, beweist ein bis heute unbekannter Brief Gessners vom 4.März 1561 an den kroatischen Polyhistor Paul Skalic (Scalighius), eine in mancherlei Beziehung ungemein interessante Persönlichkeit. Dieser Brief ermöglicht es uns, eine sehr interessante Beziehung Gessners und eine Reihe nicht unwichtiger Einzelheiten aus

${ }^{1}$ Epistolarum medicinalium libri III (ed. CASP. WoLF), Tiguri 1577. Ein vierter Band erschien Vitebergae 1584 (auch als Nachtrag in Hanharts Leben Conr. Geßners, Winterthur 1824).

${ }^{2}$ Bibliotheca medicinae practicae, II, Basileae-Bernae 1777, S. 55.

${ }^{3}$ Kurzes Handbuch der Geschichte der Medizin, Berlin 1922, S. 223.

${ }^{4}$ Im Druck wurde eine Anzahl von Briefen Gessners an Joh. Bauhin, Cuusius, Vadian, ZwINGLI u. a. herausgegeben, während sich in verschiedenen Schweizer Bibliotheken sowie in Erlangen noch unveröffentlichte Briefe GESSNERS befinden. 
dessen Leben kennenzulernen, die zum größten Teil der bisherigen Forschung verborgen geblieben sind.

Bevor wir den Text dieses Briefes wiedergeben, wollen wir einige Worte über die Person seines Empfängers vorausschicken ${ }^{5}$. PAUl Skalic wurde am Dreikönigsfest 1534 in Zagreb (Kroatien) geboren, wo sein Vater MICHAEL ein dürftiges Schulmeisterdasein führte. Schon in seiner Vaterstadt, wo der Knabe seinen ersten Unterricht genoß, fiel seine ungewöhnliche Begabung auf. Früh verwaist, kam er durch die Unterstützung einiger Wohltäter bereits mit elf Jahren nach Wien. Hier nahm sich der Laibacher Bischof Urban Textor, der sich damals am Wiener Hof als kaiserlicher Beichtvater befand, seiner an, und als eifriger Anhänger der Jesuiten (er wurde «malleus Lutheranorum» genannt!) übergab er den Jüngling den Jesuiten, damit diese für seine Erziehung sorgten. Bestimmend mag dabei gewesen sein, daß einem Gerüchte zufolge Skalic' Familie Sympathien für den Protestantismus hegte. Texror stellte den frühreifen Jüngling dem Kaiser Ferdinand vor, der an ihm Gefallen fand und sich erbot, die Kosten seines Studiums zu tragen. Schon 1547, also mit dreizehn Jahren, ist Skalic an der Wiener Universität inskribiert ${ }^{6}$ und zwei Jahre später erhält er den Titel eines Baccalaureus philosophiae. In seinem Zeugnis heißt es: «In publicis lectionibus et disputationibus specimen admirandum sui ingenii et eruditionis dedit.» ${ }^{7}$ Im Jahre 1549 erlangt Skalic den Grad eines Doktors der Philosophie, nachdem er kurz vorher Magister der Philosophie wurde, und noch im selben Jahre verläßt er Wien und begibt sich nach Bologna,

5 Ausführlich habe ich über Skalic und seine Beziehungen zur Medizin und zu Ärzten im kroatischen Ärzteblatt Lijecnicki vjesnik (Zagreb 1948, Nr. 2-3) berichtet (daselbst ist die kroatische Literatur über SkalıI angeführt). - Vgl. ferner: Acta Borussica, J. I,S. 305 und 820; SpachiUs, Nomenclator scriptorum philosophicorum atque philologicorum, Argentinae 1598, passim; CzvitTinger, Specimen Hungariae literatae, Francofurti et Lipsiae 1711, S. 331; Schelhonn, Amoenitates literariae, IX, Francofurti et Lipsiae 1726, S. 691; MANGET, Bibliotheca scriptorum medicorum veterum et recentiorum, Genevae 1731, Bd. II, T. 2, S. 165; Jöcher, Allgemeines Gelehrten-Lexikon, IV, Leipzig 1751, S. 188; Kreuzfeld, Scalichius (Berlinische Monatsschrift, 1791); Voigt, Paul Skalich, der falsche Markgraf von Verona (Berliner Kalender für 1848); KAUSLER und Sснотт, Briefwechsel zwischen Herzog Christoph von Württemberg und P.P.Vergerius, Stuttgart 1875; Elze, Die Universität Tübingen und die Studenten aus Krain, Tübingen 1877, S. 8; Elze, Trubers Briefe, Tübingen 1897, S. 62.

${ }^{6}$ Vgl. die entsprechenden Eintragungen in die Matrikel der ungarischen Nation an der Wiener Universität (Schrauf, Die Matrikel der ungarischen Nation an der Wiener Universität 1453-1630, Wien 1902, S. 200).

${ }^{7}$ Handschrift in der Wallenrodschen Bibliothek in Königsberg. 
wo er 1552 auch zum Doktor der Theologie promoviert wird. Bei seinem Examen beantwortete er 1553 Fragen und setzte alle Anwesenden durch sein ungeheures Wissen in Erstaunen.

Nun verläßt Skalic Bologna und zieht nach Rom, wo er viele Verehrer erwirbt und vielfach mit Pico della Mirandola verglichen wird. Auch hier erregt er in einigen öffentlichen Disputationen großes Aufsehen und anerbietet sich schließlich, 10447 Thesen aus allen Gebieten der Wissenschaft öffentlich zu verteidigen, doch gelingt es den Jesuiten, diese Disputation zu vereiteln. Mit einer Empfehlung des Papstes Julius III. kehrt nun Skalic nach Wien zurück, wo er dem Kaiser sein Diplom und zugleich sein Werk Conclusiones in omni genere scientiarum, das soeben (1553) erschienen war, überreicht. Bald hatte er die Gunst des Kaisers erworben, der ihn zu seinem Hofkaplan ernannte und ihm sein volles Vertrauen schenkte. Der päpstliche Legat in Wien erwirkte ihm noch die Stelle eines päpstlichen Notars.

Bis hieher verlief Skalic' Leben noch immerhin normal. Wohl ist seine Gelehrsamkeit und die Geschwindigkeit, mit der dieser frühreife Geist Karriere macht und akademische und kirchliche Würden erlangt, nicht alltäglich. Aber zu jener Zeit waren solche glänzende und rasche Aufstiege keinesfalls so ungewöhnlich, wie sie es heutzutage wären. Nun beginnt jedoch in Skalic' Leben ein neuer Abschnitt. Vermutlich unter dem Einfluß aller dieser so früh erworbenen Würden, wohl auch in der Befürchtung, seine niedrige Abkunft könnte ihm bei seinem Aufstieg hinderlich sein, beginnt nun der bisher bescheidene junge Mann sich die verschiedensten klangvollen Adelsprädikate anzueignen und unterläßt von nun an keine Gelegenheit, seine angebliche uralte adelige Abstammung hervorzuheben. Der bisherige PAulus Scalichius nennt sich von nun an «Paulus de la Scala et Hun ${ }^{8}$, Comes de Lika $^{9}$ » oder sogar «Princeps de la Scala, Comes Hunnorum ${ }^{10}$, Baro Zkradinus ${ }^{11} \gg$ usw. In den Titeln seiner Werke führt er neben seinem Namen alle seine angeblichen Adelsprädikate an, und selbst in seinen Werken finden wir an zahlreichen Stellen, ohne irgendwelche Beziehung zum übrigen Text, seinen Familienstammbaum, der seine angebliche Verwandtschaft mit fast

${ }^{8}$ Hun: willkürliche Übersetzung von Una (rechter Nebenfluß der Save in Bosnien und Kroatien) - in übertragenem Sinne auch das Gebiet um diesen Fluß.

${ }^{9}$ Lika: der südwestliche Teil Kroatiens.

${ }^{10}$ Comes Hunnorum: Herr des Gebietes um den Unafluß.

${ }^{11}$ Zkrad, alte befestigte Stadt in Kroatien, im Besitze der Frangipani, Cillier Grafen, ZRINY u. a. Im Jahre 1558 aufgelassen, 1585 von den Türken niedergebrannt. 
dem gesamten Adel Europas, ja sogar mit vielen regierenden Häusern beweisen soll. Im Jahre 1561 gab er übrigens ein eigenes Buch heraus, das den klangvollen Titel trägt Genealogia seu de antiquissima Scalichiorum sive a Scala etc. origine ab anno salutis $L X X X$ usque ad annum MDLXI, in dem sich auch SKalic' Porträt und sein Wappen (eine Leiter und ein Hirschgeweih) nebst den absurdesten Behauptungen bezüglich seiner Abstammung befinden. In jedem einzelnen seiner Werke finden wir die phantastischsten Angaben über seine Familie, daneben Abschriften uralter Urkunden und Adelsdiplome, selbst solcher aus der Zeit BELAs IV., der SKALIC' Familie angeblich wegen ihrer Verdienste im Tatarenkrieg 1263 den Adel verliehen hatte. Dieses gefälschte Adelsdiplom ließ sich Skalıc am 1. März 1555 von Kaiser Ferdinand bestätigen, bei welcher Gelegenheit der Kaiser eine Rede hielt, in der er u.a. sagte: «Ab ipsa enim infantia humaniorum liberaliorumque artium deditus studio, paucorum annorum periculo perfecit, ut peragratis variorum regnorum celebrioribus et insignioribus academiis, felicissimi ingenii solertia ac animi irrequieti industria, continuaque diligentia, difficiliorum et inter alias praecipuas quasque disciplinas excellentiorum artium cognitionem, ad unguem et ultra praeteriti saeculi hominum industriam et acumen consecutus, a doctissimis et insignibus viris, magnis in re literaria titulis et honoribus condecorari meruit.. ${ }^{12}$ Diese falsche Urkunde war die Grundlage für alle späteren Fälschungen SKaLIC'. Auf Grund dieser Urkunde konstruierte er verwandtschaftliche Beziehungen zu den Veroneser Scaligers (della Scala), zum byzantinischen Kaiserhaus der Paläologen, zu den serbischen und bosnischen Herrschern, zu den Familien Corvinus, Frangipani, zu den Herzögen von Mantua und Benevent, zu den Kurfürsten von Brandenburg usw. ${ }^{13}$

12 KukulJevic, Pavao Skalic, Zagreb 1875, S. 11.

${ }^{13}$ Es sei daran erinnert, daß in ganz analoger Weise Julius Caesar Scaliger seine Abstammung von den Veroneser Fürsten DELLA Scala nachzuweisen suchte. Sein Sohn, der berühmte Philologe Joseph Justus Scaliger, gab im Jahre 1594 eine Schrift heraus (Epistola de vetustate et splendore gentis Scaligerae), in der er sich bemühte, diese Behauptung durch Beweise zu stützen. Auf diese Schrift antwortete Kaspar Schoppe (SciopPIUS) mit einem $1607 \mathrm{zu}$ Mainz erschienenen Pamphlet Scaliger hypobolomaeus, hoc est elenchus epistolae J. Burdonis pseudo-Scaligeri, in dem er die Fabel von Scaligers fürstlicher Abstammung zu zerstören trachtete. J.J.ScALIGER antwortete seinerseits auf dieses Pamphlet in einer anonymen Schrift Confutatio stultissimae Burdonis fabulae (Lugd. Bat. 1609). Es ist bemerkenswert, daß Scaliger in seiner ersten Schrift auch SKalic' Familie zu den Nachkommen der Veroneser Scaliger zählt: «Comites de Lika, ex quibus prognati sunt Philippus et Bartholomaeus Scaligeri, qui pulsum regno a 
Solche grobe und dreiste Fälschungen konnten auf die Dauer nicht unentdeckt bleiben. Dazu kam noch, daß SKaLIc wegen eines öffentlichen Angriffs auf die Irrtümer der katholischen Kirche die Mißgunst der Jesuiten erregt hatte. Seine früheren Beschützer begannen sich langsam zurückzuziehen, worauf sich Skalic den Protestanten näherte. Er verband sich mit Johann Sebastian Phauser, dem Prediger des jungen Königs MaximiLIAN, der ein Feind der Jesuiten und Anhänger des Protestantismus war. Seinem Einfluß bei Maximilian hatte Skalic es zu verdanken, daß der König ihn in seinen Schutz nahm. Aber Skalic' Zuwendung zum Protestantismus erschütterte Ferdinands Vertrauen vollends, um so mehr, als er bereits von SKalic' Fälschungen erfahren hatte. Der Kaiser enthob Skalic seines Amtes als Hofkaplan, und dieser schloß sich jetzt nur noch enger an die protestantische Partei Maximilians an. Die Folge davon war, daß SKalic schließlich Wien verlassen mußte: 1577 ging er zuerst nach Preßburg und von da nach Prag, dann nach Regensburg und schließlich nach Tübingen. Mit einem Empfehlungsschreiben MaximiLians erschien er am Hofe des Herzogs Christoph von Württemberg, wo er eine größere Anzahl seiner Landsleute antraf (Petrus Paulus Vergerius, Matthias Flacius Illyrigus, Georg Dalmatin, Primus Truber, Stephan Konsul u. a.), die als Anhänger des Protestantismus ausgewandert waren und hier eine Zufluchtsstätte gefunden hatten. Hier lernte Skalic auch den verdienstvollen Freiherrn von UNGNAD, den ehemaligen Befehlshaber der kroatischen Militärgrenze, kennen, der in Urach eine Buchdruckerei gegründet hatte, wo die kroatischen und slowenischen protestantischen Bücher gedruckt wurden.

Hier in Tübingen trat SKalic offen zur protestantischen Kirche über. Auch hier behauptete er, ein Opfer des Protestantismus zu sein, und erklärte, er wäre ein Nachkomme der Fürsten Scaliger von Verona, der wegen

Tartaris Regem Hungariae Belam virtute sua in integrum restituerunt: ac propterea a Rege Bela Castro Zkrad in Croatia, regione Hun in Hungaria donati sunt, ac insuper Commilitones Regis vocati, qui sunt tanquam Palatini Regni» (vgl. J.J.SCALIGER, Epistolae, Lugd. Bat. 1627, S. 12). Scaliger, der das Unagebiet (Regio Hun) fälschlich für ein ungarisches Gebiet hält, folgert daraus, daß die Familie Hunyady (Johannes Hunyady und sein Sohn König Matthias Corvinus) mit den Scaligers verwandt sei (ibid.). Dagegen äußert er sich in der zweiten Schrift über SKalic folgendermaßen: «Paulus Scalichius, qui se pro Michaelis filio gerebat, homo Slavus erat, in Carnis natus, in Croatia educatus, in Germania institutus. An de genere suo mentitus sit, id neque scimus, neque confutamus, neque nostra refert» (J.J.Scaliger, Opuscula varia, Francofurti 1612, S. 102). 
seines Beitritts zum Protestantismus von seinen Gütern in Italien und Kroatien vertrieben worden sei. Er nennt sich jetzt «Comes Hunnorum et Marchio Veronae» oder auch «Totius terrae ex utraque parte Hun nec non Zkrad atque Veronae etc. Dominus et Comes» usw. Auch den Freiherrn von UNGNAD wußte er durch verschiedene Lügen und Fälschungen vollkommen für sich zu gewinnen, so daß ihm UNGNAD sogar versprach, ihm zur Wiedererlangung seiner Besitztümer zu verhelfen. Ihm hatte es SKaLIC auch zu verdanken, daß er zum Professor an der Universität Tübingen ernannt wurde, wo er eine Zeitlang theologische Vorlesungen hielt.

SKaLIC' sehnlichster Wunsch war es indessen, wieder nach Wien zurückkehren zu können. Dazu sollten ihm auch UngNads Beziehungen zu König Maximilian verhelfen. Auch selbst wandte er sich mehrfach an den König, dem er übrigens seine kroatische Übersetzung des protestantischen Katechismus übersandte. Auch haben sich drei Briefe Maximilians an Skalic erhalten, in denen er u. a. die Schwierigkeiten schildert, auf welche die Erfüllung von SKalic' Bitte stößt. Schließlich verscherzte sich SKalic auch Maximilians Gunst, indem er, entgegen dessen ausdrücklicher Ermahnung, seinen Stammbaum an den Kaiser sandte. Von nun an versagte Maximilian Skalic jede weitere Unterstützung, so daß Skalic seinen Plan, nach Wien zurückzukehren, endgültig aufgeben mußte.

So entschloß er sich 1561, Tübingen zu verlassen und nach Königsberg zu ziehen, wohin ihn Herzog Albreght von Preußen auf Ungnads Veranlassung berufen hatte. Nach dreijährigem Aufenthalt in Tübingen machte sich SKalic im November 1561 auf den Weg und traf gegen Ende des Jahres in Königsberg ein. Herzog Albrecht, damals ein Mann von siebzig Jahren, war ein Freund aller Gelehrten, besonders derjenigen, die wegen des Protestantismus verfolgt wurden ${ }^{14}$. Er ernannte SKalic zu seinem Minister mit einem lebenslänglichen Jahresgehalt von 1000 polnischen Gulden und stellte ihm einen Palast als Wohnung zur Verfügung. Auf Grund seiner gefälschten Dokumente gelang es SKaLIC, den greisen Herzog, für dessen Verwandten er sich übrigens ausgab, ganz für sich zu gewinnen, was natürlich bald bei den übrigen Höflingen Mißgunst erregte. Um SKaLIC zu seinen verlorengegangenen Familiengütern zu verhelfen, gab ihm der Herzog Empfehlungsschreiben für den Kaiser und für König Sigismund von Polen.

${ }^{14}$ Auch die protestantischen Emigranten aus Kroatien und Krain fanden in Albrecht einen mächtigen Beschützer und Förderer. War er es doch, der UNGNAD die Geldmittel zur Gründung der protestantischen Druckerei in Urach zur Verfügung stellte. 
Anfangs 1562 ging SKalIC sogar persönlich an den polnischen Hof, wo er ehrenvoll empfangen wurde und wo seine Gelehrsamkeit einen tiefen Eindruck machte. Voll der schönsten Hoffnungen bat er nach seiner Rückkehr den Herzog, zu seinen Gunsten an König Maximilian zu schreiben, was der Herzog auch tat; aber MAXIMILIAN antwortete nicht.

Allmählich erregte Skalic' Ausnahmsstellung am preußischen Hof Neid und Unzufriedenheit. Einige seiner Gegner griffen ihn wegen seiner Lehren an, indem sie behaupteten, seine Lehren widersprächen dem Geiste des Protestantismus und könnten dem Ansehen der Königsberger Universität schaden. Ein Verbot von Seiten der Universität an die Studenten, SKalic' Vorlesungen anzuhören, rief bei diesen jedoch Empörung hervor.

Im Jahre 1563 veröffentlichte Skalic ein Gespräch mit dem venetianischen Gesandten am polnischen Hof und fiel deshalb beim polnischen Hof in Ungnade. Er ahnte damals gar nicht, daß diese seine Indiskretion für ihn verhängnisvoll werden sollte. Auch in Preußen nahm die Zahl seiner Neider und Feinde von Tag zu Tag zu. Um sich mit einem Kreis von Freunden und Beschützern zu umgeben, berief er eine Anzahl Gelehrter nach Königsberg, die das Gegengewicht zu den immer zahlreicher werdenden Gegnern bilden sollten ${ }^{15}$. Nun traf ein Brief der polnischen Königin KathaRINA ein, die dem Herzog das Ergebnis ihrer Nachforschungen über Skalic mitteilte und dabei eine Anzahl von Fälschungen entdeckt hatte. Ihr Vater, Kaiser Ferdinand, fügte auch einen Brief bei, in dem er SKalic entlarvt und u.a. schreibt: «... er ist der hoffärtigste Mann, den ich mein lebenlang nur kennengelernt, dazu gar ein Lügner und gar ungesundig» ${ }^{16}$. Selbst König Maximilian, der einstige Beschützer SKalic', fügte ein Schreiben in gleichem Sinne bei. Wieder gelang es SKaLIC, den Herzog zu beruhigen und alle diese Briefe als die Folge von Verleumdungen seitens seiner Feinde hinzustellen. Wieder fälschte er Stammbäume, die auf den Herzog einen solchen Eindruck machten, daß er SKaLIC das Schloß Kreuzburg schenkte (1564). Von nun an nennt sich SKaLIC auch noch «Cruciburgi in Borussia Dynasta ac perpetuus Borussiae Ducis consiliarius».

Der mächtigste Gegner Skalic' am preußischen Hof war Albrecht Truchseß von Wetzhausen, der alles unternahm, um Skalic' Fälschungen bekanntzumachen. Skalic klagte ihn jedoch wegen Verleumdung ein und

${ }^{15}$ Er berief u. a. Michael Scrinius aus Danzig, Johann Campinge aus Grönningen und HeinRich Zell aus Straßburg.

${ }^{16}$ Vgl. ELze, Trubers Briefe, S. 62. 
das Gericht entschied, Wetzhausen habe innerhalb eines Jahres die Beweise vorzulegen. Durch eine Reihe von Intrigen wurde jedoch die rechtzeitige Ankunft des Beweismaterials vereitelt und Herzog Albrecht sprach ein Urteil zugunsten Skalic' aus. Dieses Urteil rief nicht nur in ganz Preußen große Erregung hervor, sondern veranlaßte auch den polnischen König Sigismund II. August, eine Kommission nach Königsberg zu senden, um eine Untersuchung gegen SKalic einzuleiten. Als die Kommission am 25. August 1566 in Königsberg eintraf, fand sie SKaLIC nicht mehr vor. Dieser war nämlich inzwischen als herzöglicher Gesandter nach Frankreich gegangen, um eine Prinzessin für Albrechts Sohn zu werben. Er kam zuerst nach Danzig, wo er wieder Urkunden zu seinen Gunsten fälschte, ging dann nach Thorn und Posen und kam schließlich über Berlin, Wittenberg, Halle, Erfurt, Gotha und Frankfurt nach Paris. Inzwischen verurteilte ihn die Kommission zur Landesverbannung und erklärte ihn als vogelfrei.

Aus Paris begab sich Skalic nach Münster, wo er wieder zur katholischen Kirche zurückkehrte und zum Kanonikus ernannt wurde. Seine Schriften aus dieser Zeit sind durchwegs im Sinne des Katholizismus geschrieben. Um nach Preußen zurückkehren zu können, wandte er sich wieder brieflich an Herzog Albrecht, doch dieser antwortete ihm nicht. Nach dem Tode Sigismunds II. Augusts (1573) erlaubte der neue polnische König Heinrich von Anjou Skalic die Rückkehr nach Polen und Preußen und versprach ihm die Rückgabe der konfiszierten Güter. Skalic kam auch tatsächlich nach Königsberg, mußte aber feststellen, daß der Nachfolger des inzwischen (1568) verstorbenen Herzogs Albrecht, AlBRECht Friedrich, keine Neigung zu ihm besaß. Enttäuscht verließ er wieder Preußen, um nach Münster zurückzukehren. Die Rückreise führte ihn über Danzig, wo ihn im Sommer 1575 der Tod ereilte.

Der äußere Lebenslauf dieses merkwürdigen Mannes ist wahrlich wenig geeignet, unser Vertrauen zu erwecken. Und dennoch würden wir SKalic unrecht tun, wenn wir ihn als gewöhnlichen Abenteurer bezeichnen wollten $^{17}$. Seine Gelehrsamkeit, Klugheit, Wohlerzogenheit und Höflichkeit verschafften ihm überall Freunde und Verehrer; ohne diese Eigenschaften hätte sich sein Leben freilich ganz anders gestaltet. Die einzigen Schwächen seines Charakters waren seine Eitelkeit und seine Ruhmsüchtigkeit, die ihn

${ }^{17}$ KUKULJEvic, der kroatische Biograph SkaLIc', nennt ihn ganz unzutreffenderweise den «Cagliostro des 16. Jahrhunderts.» 
zu jenen Fälschungen verleiteten, durch die sein Bild verdüstert wird. Wohl weist sein äußerliches Leben eine Fülle von abenteuerlichen Zügen auf und doch hinterließ uns dieser Mann eine große Anzahl von Werken, die von seiner großen Gelehrsamkeit und seiner erstaunlichen Vielseitigkeit Zeugnis ablegen. Selbst diejenigen, die von seinem Charakter die schlechteste Meinung hatten, mußten zugeben, daß er ein ganz bedeutender Geist war. So charakterisiert ihn Joseph Justus Scaliger mit den Worten: «Paulus Scalichius qui se disoit estre de la Maison des Scaligers, et n'en estoit pas pourtant, avoit grand esprit.. ${ }^{18}$

Die Zahl der von SKalic teils in lateinischer, teils in deutscher Sprache verfaßten Werke wird auf etwa 25 geschätzt. Ein großer Teil davon ist rein polemischen Inhalts und bezweckt den Nachweis von SKaLIC' uradeliger Abstammung. Von den übrigen Werken verdienen Erwähnung:

1. Encyclopaediae, seu orbis disciplinarum tam sacrarum quam profanarum epistemon (Basileae 1559),

2. Satyrae philosophicae sive miscellaneorum tomus I. (Regiomonti Borussorum 1563),

3. Judicium de praecipuis sectis nostrae aetatis. De proprietatibus Antichristi et de extremo judicio (Coloniae 1569),

4. Miscellaneorum de rerum causis et successibus et de secretiore quadam methodo, qua eversiones omnium universi orbis et futurorum series erui possint libri VII (Coloniae 1570),

5. Certissima methodus, qua homines palantes et erroribus turbulentis impliciti ad viam veritatis revocandi et ad beatitudinem consequendam promonendi veniant, contra Centurias evangelicae veritatis Joh. Nasi minoritae (Coloniae 1570),

6. Dialogus de lyra (Coloniae 1570),

7. Vaticiniorum et imaginum Joachimi Abbatis Florensis Calabriae et Anselmi Episcopi Marsichani super statu summorum Pontificum Rhomanae Ecclesiae, contra falsam, iniquam, vanam, confictam et seditiosam cuiusdam Pseudomagi, quae nuper nomine Theophrasti Paracelsi in lucem prodiit, pseudomagicam expositionem, vera certa et indubitata explanatio (Coloniae 1570),

8. Miscellaneorum tomus II, sive catholici Episthemonis contra quandam corruptam ac depravatam encyclopaediam libri $X V$, quibus versus orbis

${ }^{18}$ Scaligerana, Cologne 1695, S. 352. 
tam sacrarum quam profanarum disciplinarum omnis sectarum et philosophiae catholicae doctrinae declaratur (Coloniae 1571),

9. Loci communes theologici (Coloniae 1571). ${ }^{19}$

Wie aus den Titeln ersichtlich, werden in diesen Werken hauptsächlich theologische Fragen behandelt und zwar, bezeichnenderweise für deren Verfasser, teils im Geiste des Protestantismus, teils des Katholizismus. Aber Skalic beschränkt sich keinesfalls auf die Theologie allein, sondern es gibt kaum ein Wissensgebiet jener Zeit, das ihm fremd ist und das in seinen Schriften nicht irgendwie behandelt wird. Besonders sein Hauptwerk Encyclopaediae enthält sozusagen das gesamte Wissen seiner Zeit und stellt einen der erfolgreichsten enzyklopädischen Versuche des 16.Jahrhunderts dar. In der Geschichte der Enzyklopädien spielt dieses Werk insoferne eine bedeutsame Rolle, als es das erste Werk dieser Art ist, das sich im Titel «Enzyklopädie» nennt ${ }^{20}$. Einen weiten Platz nimmt in SKaLIG' Schriften die Behandlung okkultistischer Fragen ein, aber er zeigt sich auch in naturwissenschaftlichen und namentlich in medizinischen Fragen wohlunterrichtet. Bedeutungsvoll ist sein Encomium medicinae ${ }^{21}$, das selbst Haller als medizinische Schrift erwähnt, nicht ohne dessen Verfasser als «inconstans homo» zu charakterisieren ${ }^{22}$. Wir werden auf diese Schrift noch zu sprechen kommen. Viel Medizinisches findet sich auch in den übrigen Kapiteln von SKalic' Enzyklopädie sowie an einigen Stellen seiner übrigen Werke. Seine oben erwähnte, gegen Paracelsus gerichtete Schrift hat wohl mit Medizin nichts zu tun, sondern ist ein Angriff auf den «Magier» Paracelsus und dessen Wunderbare Weissagung vom Papsttum (in seiner Practica gemacht auff Europen), die SKaLIG zu widerlegen sucht.

Selbst mathematische und musikologische Fragen (letzteren widmete er seine Schrift Dialogus de lyra) beschäftigten SKaLIC und wurden von ihm eingehend behandelt. Am meisten fesselten ihn jedoch okkulte Dinge und die Magie scheint zu seinen Lieblingsbeschäftigungen gehört zu haben. Er verkehrte mit Geistern, besaß magische Münzen und Ringe, verfaßte auch

${ }^{19}$ Eine Bibliographie von Skalic' Werken findet sich bei Simmler (Epitom. Biblioth. Conr. Gesneri, Tiguri 1574, S. 547), ferner in den angeführten Werken von Spachius, CzvirTINGER, SchelhorN, Jöcher, KuKUlJEvic usw. Keine von diesen Zusammenstellungen kann indessen Anspruch auf Vollständigkeit erheben.

${ }^{20}$ Vgl. Brockhaus' Konversationslexikon (13. Auflage) und Encyclopaedia Britannica s. v. «Enzyklopädie» bzw. «Encyclopaedia».

${ }^{21}$ Encyclopaediae, S. 725-37.

${ }^{22}$ Bibliotheca medicinae practicae, tom. II, Basileae/Bernae 1777, S. 142. 
ein kabbalistisches Gebet zum Zwecke der Teufelsaustreibung, behauptete u. a. die Jakobsleiter gesehen zu haben, über die man in den Himmel aufsteigen könne usw. Diesen groben Unfug brandmarkte Johannes WeYer in seinem berühmten Werk De praestigiis daemonum ${ }^{23}$, wo er SKallc persönlich angriff, worauf dieser seinerseits eine scharfe Entgegnung verfaßte ${ }^{24}$, in der er, gestützt auf eine Anzahl von Autoritäten, seine Behauptungen zu bekräftigen suchte.

Skalic' medizinische Kenntnisse, die an verschiedenen Stellen seiner Werke zum Ausdruck kommen ${ }^{25}$, müssen wir angesichts der Tatsache, daß er niemals Medizin studiert hatte, geradezu imponierend nennen. In seinen medizinischen Anschauungen ist Skalic ein Anhänger Galens, wie die Mehrzahl der Ärzte jener Zeit. Er vertritt die Lehre von den vier Elementen, den vier Säften und der sogenannten «vis animae» mit ihren dreierlei Äußerungsarten (vegetativa, sensitiva et rationalis). Entgegen der Lehre Aristoteles', vertritt er den Standpunkt Galens, daß die Nerven dem Gehirn und nicht dem Herzen entspringen, wobei er motorische (harte) und sensible (weiche) Nerven unterscheidet. Er beschreibt ferner die einzelnen physiologischen Funktionen, besonders ausführlich den Atmungsmechanismus, dessen Zweck seiner Ansicht nach die Abkühlung des Herzens ist. Seine Kenntnisse des Blutkreislaufs entsprechen den damaligen allgemeinen Kenntnissen, doch verwirft er den Begriff der "vis pulsifica» und vertritt die Meinung, daß die Zusammenziehung und Erschlaffung des Herzens sich in die Arterien fortpflanzen und daselbst den Puls hervorrufen. Sкalic unterscheidet die theoretische Medizin (Physiologie, Ätiologie und Semiologie) von der praktischen (Hygiene und Therapie). Als Krankheitsursachen kommen für ihn in Frage: Schädlichkeiten in der Atmungsluft, in der Nahrung und in den Getränken, Unregelmäßigkeiten in der Ruhe und Bewegung, im Schlafen und Wachen und schließlich seelische Erregungen.

Den Sinnesorganen und den höheren seelischen Funktionen widmet SKalic ausführliche Erörterungen, wobei ein gewisses Bestreben, die psychischen Phänomene materialistisch zu erklären, auffällt. Ebenso fällt uns das (allerdings primitive) Bestreben auf, die einzelnen seelischen Funktionen in den einzelnen Gehirnpartien zu lokalisieren. Erwähnenswert er-

${ }^{23}$ Im Nachtrag Liber apologeticus de pseudomonachia daemonum.

24 Miscellaneorum tomus II, S. 218.

${ }^{25}$ Die hauptsächlichsten Stellen befinden sich in Encyclopaediae, S. $49 \mathrm{ff}$., $549 \mathrm{ff}$. und $725 \mathrm{ff}$. 
scheint Skalic' Behauptung, der Mensch erreiche im Schlaf dasjenige, was er im wachen Zustand ersehnt ( «Somnians plerumque ea quae vigilans voluit, habet»), eine Erkenntnis, die dreieinhalb Jahrhunderte später eine der Grundideen der Freudschen Psychoanalyse darstellt.

Besonders interessant ist SKaLIG' Encomium medicinae, ein Abschnitt seines Encomium (siehe oben!). Nach einer kurzen Darstellung der geschichtlichen Entwicklung der Medizin, wobei die einzelnen Schulen und ihre Hauptvertreter aufgezählt werden, kommt SKalic zu dem Ergebnis, daß die Medizin sowohl eine Wissenschaft als auch eine Kunst sei. Ihr Ziel ist die Erforschung und Bekämpfung derjenigen Faktoren, die dem Menschen schaden. Die Idealgestalt des Arztes ist ihm Hippokrates, dessen Auffassungen von Gesundheit und Krankheit er kurz darstellt. Besonders wichtig erscheint ihm die Entfernung der schädlichen Säfte aus dem Organismus, zu welchem Zweck ihm der Aderlaß als das geeignetste Mittel erscheint. Für das ärztliche Handeln ist die Kenntnis der Affekte, der Temperamente, der einzelnen Diathesen, der Puls- und Fieberarten von größter Wichtigkeit. Überhaupt ist die Diagnostik nach SKALIG' Ansicht die Grundlage der Medizin. Indem er eine Reihe der bedeutendsten Ärzte aller Zeiten und Völker aufzählt und ihre Verdienste würdigt, kommt er zu dem Schluß, daß die Heilkunde wirklich ein Lob verdiene, da sie keineswegs steril sei, sondern den Menschen zum Segen und ihren Schöpfern zur Ehre gereiche.

Schließlich wollen wir noch eine Stelle in SKaLIc' Schriften erwähnen, die vom medizinischen Standpunkt interessant ist. In seiner Schrift Satyrae philosophicae befindet sich auf Seite 149 ein Abschnitt unter dem Titel «De pulvere Tritemii, tum eius adversus varios morbos, videlicet, stomachi, cerebri, oculorum, memoriae, humorum pectoris, nauseae, nimiae pinguedinis, epilepsiae, apoplexiae, calculi, chiragrae, podagrae, sciaticaeque, admiranda efficatia». In diesem, dem Herzog Albregrt von Preußen gewidmeten Abschnitt befindet sich ein langatmiges Rezept eines angeblich von Johannes Tritheim erfundenen Pulvers, das eine Art Panazee darstellt. Hernach folgt ein ausführlicher Dialog zwischen SKaLIC und den herzöglichen Leibärzten, wobei er die Vorzüge und wunderbare Wirkungsweise dieses Pulvers hervorhebt.

Wie in allen seinen übrigen Werken, zitiert Skalıc auch in den medizinischen eine große Anzahl von Autoren und beweist auf diese Art seine große Belesenheit, der er ja schließlich allein seine medizinischen Kenntnisse verdankt. Was SKalic an medizinischen Schriften verfaßt hat, ist nicht wertloser als das meiste, was wir aus der medizinischen Literatur jener 
Tage kennen. Es ist eben medizinische Buchweisheit, die im 16. Jahrhundert trotz Paracelsus und Vesal noch immer den Großteil des medizinischen Schrifttums bildet.

Unter den Männern, zu denen SkaLIc Beziehungen unterhielt, finden wir denn auch eine Anzahl von Ärzten, die ihn als Menschen und Gelehrten schätzten. Unter diesen wäre Petrus Maynardus, Professor der Medizin in Padua, zu erwähnen ${ }^{26}$, der besonders für SKalic' kabbalistische Schriften reges Interesse zeigte ${ }^{27}$, ferner der berühmte Tübinger Arzt und Geschichtsforscher Oswald Gabelchover (Gaebelkhouer) ${ }^{28}$, der zu Skalic' begeistertsten Anhängern gehörte und ihm sogar ein Gedicht widmete, das in seiner Genealogia abgedruckt ist. Enge Beziehungen bestanden auch zwischen Skalic und dem Leibarzt des Bischofs von Münster, Dr. Gerhard Martell, dem Skalic 1570 ein Kapitel seines Werkes Miscellaneorum de rerum causis widmete ${ }^{29}$. Auch einen Leibarzt des Herzogs Albrecht von Preußen, namens Ganitzius, erwähnt Skalic anerkennend («mihi tamquam frater carissimus») ${ }^{30}$. Vermutlich bestanden auch Beziehungen zwischen Skalic und dem berühmten Leonhard Fughs, den Skalic wohl lobend erwähnt ${ }^{31}$, ohne jedoch etwas über gegenseitige persönliche Beziehungen anzudeuten. Da die beiden zu gleicher Zeit in Tübingen wirkten und an der dortigen Universität lehrten, außerdem auch beide die Gunst des Herzogs von Württemberg genossen, ist eine persönliche Bekanntschaft der beiden mehr als wahrscheinlich. Über SKalic' Beziehungen zu Conrad Gessner, Jakob Schegk und Venerand Gabler werden wir noch ausführlich sprechen. Auch unter SKaLIc' Gegnern finden wir einige Ärzte: so griff ihn 1563 der Königsberger Professor der Medizin und herzögliche Hofarzt Dr. Matthias Stojus ${ }^{32}$ wegen seiner theologischen Lehren an und zur gleichen Zeit beschuldigte ihn auch Dr. Simon Tiтıus, gleichfalls Professor der Medizin in Königsberg ${ }^{33}$, durch seine falschen Lehren die Jugend zu verderben. Von Skalic' Streit mit Johannes Weyer war bereits die Rede.

${ }^{26}$ Vgl. Biographisches Lexikon der hervorragenden Ärzte, Bd. IV, S. 136.

${ }^{27}$ Vgl. Encyclopaediae, S. 421.

${ }^{28}$ Vgl. Biographisches Lexikon der hervorragenden Ärzte, Bd. II, S. 652.

29 «Epistola qua omnes abditae artes et scientiae perstringuntur et perfectissima ratio prophetandi et miracula operandi traditur» (S. 265).

${ }^{30}$ Vgl. «De pulvere Tritemii» (Satyrae philosophicae, S. 149).

${ }^{31}$ Encyclopaediae, S. 734.

${ }^{32}$ Vgl. Biographisches Lexikon der hervorragenden Ärzte, Bd. V, S. 438.

${ }^{33}$ ibid. S. 595. 
Und nun wollen wir uns mit dem eingangs erwähnten Brief Gessners an SKalic befassen, der uns einiges über die gegenseitige Beziehung der beiden Männer sagt. Dieser Brief ist in SkaLIc' Werk Satyrae philosophicae ${ }^{34}$ abgedruckt und lautet:

Illustri et generoso viro D. Paulo Scalichio de Lika. Comiti Hunorum, \& Baroni castri Zkrad. Doctori Theologiae absolutissimo, Domino suo honorando.

S. Brevissima tua sed suavissima Epistola (vir genere et variis virtutibus nobilissime) nono Octobris ad me data: eam quem de tuo candore, \& erga me amorem opinionem merito quidem tuo, conceperam, plene confirmavit. Gaudeo officium meum in mentione tui facta, cum pisces Maximiliano Regi offerrem, non ingratum tibi fuisse, animus quidem tuus magnorum mihi numinum instar est. Hoc peto a praestantia tua, ut quandocunque commode fieri poterit, per literas ad amicum aliquem Maximiliano familiarem sciscitetur, nunquid liber a me missus ad Regem, ipsi oblatus sit, quomodo erga ipsum afficiatur. Quamvis enim ante menses fere VII. librum pulchre ligatum miserim, \& ab eo temporis ad Caesaris medicos aliosque scripserim, nullum responsum tuli. Itaque nihil gratius hoc tempore mihi praestiteris, quam si ut hac de re certior fiam effeceris. Autumno superiore mecum fuit eruditus et bonus juvenis G. Keutherus Prutenus, qui multa mirifice de doctrina \& aliis virtutibus tuis praedicabat (is ubi nunc sit miror, \& quod tam diu nihil ad nos scribat). Addebat familiam tuam Scaligeris gentilem esse: quod equidem intelligere mihi volupe erat. Veneror enim Iulii Caesaris Scaligeri, summi \& absolutissimi omnigena doctrina viri, quotquot extant lucubrationes, \& ante paucos dies a doctissimo eius filio (Sylvio nomine) literas amoris plenas accepi, quibus me ad amicitiam mutuam invitat: ac simul Rob. Constantini, juvenis Graece Latineque eruditissimi, epistolam, qua libros aliquot a defuncto felicis memoriae Scaligero, sibi legatos scribit: eosque se curaturum ut brevi edantur: Iamque justum ipsius de Poetica commentarium Genevae excusum esse. Hoc te scire volui. Sed quoniam amicorum est non jucunda, solum communicare, sed prorsus omnia, juxta proverbium: non potui non aperire tibi, quanque

${ }^{34}$ Dieses Werk wurde 1563 in Königsberg in der Offizin des herzöglichen Buchdruckers Johannes Daubmann gedruckt. Es stellt einen Sammelband dar, wie dies ja auch im Untertitel («Miscellaneorum tomus II») betont ist. Ein Kapitel dieses Buches trägt den Titel «Apologiae» und enthält eine große Anzahl von Dokumenten (Urkunden, Privilegien, Zeugnisse, Briefe usw.), die durchwegs eine Verherrlichung Skalic' und seiner Abstammung bezwecken. Unter diesen Briefen befindet sich auf S. 636 auch GessNers Brief. 
invitus, quod nuper quidem ex literis nescio cujus nec ad quem, transcriptum, mihi ostendit, ego ut te admonere possem, excepi, ut legas in charta his literis inserta. Haec per Sebastianum Polonum Peclinum, quem tibi commendo, scribenda ad te habui. Plura de rebus nostris ex ipso cognosces. Vale.

Tiguri Martii die 4. Anno 1561. Raptim.

$$
\begin{gathered}
\text { Amplitudinis tuae } \\
\text { studiosissimus }
\end{gathered}
$$

Con. Gesnerus Philiatros.

Clarissimos Viros D.D. Scheccium \& Gablerum plurimum meo nomine salutare cupio. D. Scheccium delectari audio Scaligeri libris. Quamobrem quae de eo scripsi communicabis: \& praeter librum de Poetica excusum: Opus Nobilium exercitationum brevi subiturum praelum: deinde etiam commentarios in aliquot Theophrasti \& Aristotelis de plantis \& animalibus (quod ad caussas praecipue) libros. Eorum editionem me quoque promoturum; idque rogitatu Constantini: cuius thesaurum linguae Graece longe copiosissimum brevi dabit Geneva.

Betrachten wir zuerst den Inhalt dieses Briefes. Gessner bestätigt den Empfang eines Briefes, den er am 9. Oktober 1560 von Skalic erhalten hatte. Er freut sich, daß Skalic nichts dagegen einzuwenden hatte, daß er (Gessner) unter Berufung auf Skalic dem König Maximilian irgendwelche Fische (?) dargeboten hatte. Nun bittet er Skalic, durch irgendeinen Freund, der dem König nahesteht, nachfragen zu lassen, ob der König das ihm von Gessner übersandte Buch erhalten habe. Obgleich er bereits bei den kaiserlichen Leibärzten nachgefragt habe, habe er bisher keine Antwort erhalten. Nun folgt ein Bericht über einige gemeinsame Bekannte, und zwar zunächst über einen gewissen KeUTHER, der augenblicklich aus Gessners Gesichtskreis verschwunden ist und der ihm im vergangenen Herbst viel wunderbares über SkaLIC' Gelehrsamkeit und dessen verwandt schaftliche Beziehungen zu den Scaligers berichtet hat. Gessner lobt besonders Julius Caesar Scaliger, mit dessen Sohn Sylvius er einen freundschaftlichen Briefwechsel unterhält. Weiter erwähnt er einen gelehrten Jüngling namens Robert Constantin, der demnächst einige Werke des verstorbenen Julius Caesar Scaliger herausgeben wird. Sein Kommentar zur Poetik sei soeben in Genf erschienen. Auch mit ihm stehe Gessner in brieflichem Verkehr.

Es folgt nun eine geheimnisvolle Allusion auf gewisse Gerüchte über Skalic, die Gessner zu Ohren gekommen sind und die er, um Skalic zu ermahnen, diesem auf einem separaten Blatt mitteilt. 
Diesen Brief übersandte Gessner durch einen Polen, Sebastian PegLINUS, den er SKalic empfiehlt und von dem dieser noch weiteres erfahren werde. In der Nachschrift sendet Gessner Grüße an Schecci us und GabLER und bittet Skalic, Scheccius mitzuteilen, daß demnächst einige Werke Julius Caesar Scaligers auf Gessners Veranlassung im Druck erscheinen werden, desgleichen Constantins griechisches Wörterbuch.

So kurz und scheinbar nichtssagend dieser Brief ist, sein Inhalt gestattet uns dennoch mancherlei Schlüsse, die nicht ganz belanglos sind und die manche Momente aus Gessners Leben in einem neuen Licht darstellen.

Vor allem sehen wir, daß Gessner daran gelegen ist, mit SKaLIG' indirekter Hilfe Beziehungen zu König Maximilian anzubahnen. Dieses Bestreben Gessners war bisher nicht bekannt. Allerdings wählte er dazu einen sehr ungeeigneten Vermittler, da SkaLIC um diese Zeit sich bereits die Gunst Maximilians verscherzt hatte. Früher wäre das freilich möglich gewesen, hatte doch Maximilian noch 1558 Skalic versprochen, Lälius Socin und Johannes Oporin mit allen Ehren zu empfangen ${ }^{35}$. Die Berufung auf Skalic mag ja auch der Grund gewesen sein, daß sich MaximiLIAN für das zugesandte Buch nicht bedankt hatte. Gessner erwähnt in seinem Brief, er hätte dem König irgendwelche Fische (pisces) dargeboten. Höchstwahrscheinlich meint er damit sein Fischbuch, den 4.Band seiner Historia animalium, der den Untertitel «Pisces» trägt und der 1558 gedruckt wurde. Die folgende Erwähnung eines schön gebundenen Buches, das er dem König übersandt hatte, würde ja damit übereinstimmen. Daß dieses Buch dem Kaiser Ferdinand gewidmet war, mag ein weiterer Grund für die kühle Aufnahme seitens Maximilians gewesen sein.

Wer mögen die kaiserlichen Leibärzte gewesen sein, bei denen sich Gessner erkundigte, ob der König sein Buch erhalten habe? In Frage kommen Crato von Krafftheim, Matthioli, Alexandrinus von Neustein und Amerfort, zu denen Gessner Beziehungen unterhielt. Namentlich die zwei letzteren waren es, die ihn bewogen hatten, den 4.Teil seines zoologischen Werkes dem Kaiser zu widmen. Höchstwahrscheinlich wird Gessner mit dieser Mission auch seinen Freund Crato von Krafftheim

${ }^{35}$ In einem Brief vom 25. August 1558 schreibt Maximilian aus Wien an Skalic: «Nos etiam eundem Sozinum, nec non JoANNEM OPORINum postquam nos convenerint et quos nobis commendatos esse petis, Regia clementia complecti et qua tandem commoda occasione poterimus, eos clementer promovere dignabimur» (Satyrae philosophicae, S. 533). - Über LäLIUS Socin, den Neffen Faustus Socins, vgl. Illgen, Vita Laelii Socini (1814). Über OpORIN siehe weiter unten! 
betraut haben, der noch zu Ferdinands Lebzeiten in ein vertrauliches Verhältnis zu Maximilian getreten war $^{36}$.

Der gelehrte Jüngling G. Keuther, den Gessner erwähnt, ist nicht bekannt. Durch ihn erfuhr Gessner von der angeblichen Verwandtschaft Skalic' mit der Gelehrtenfamilie Scaliger, von der natürlich keine Rede sein kann, die sich aber aus SKalic' Stammbaumfälschungen zwanglos ergibt $^{37}$. Gessners Anerkennung der Verdienste des im Jahre 1558 verstorbenen Julius Caesar Scaliger ist vollkommen begründet. Weniger bekannt ist seine Beziehung zu dessen Sohn Sylvius. Dieser war der älteste Sohn Julius Caesar Scaligers, um zehn Jahre älter als sein berühmter Bruder Joseph Justus, und starb 1585 im Alter von fünfundfünfzig Jahren $^{38}$. Obwohl er selbst literarisch nicht tätig war, ist sein Name dennoch in der Literaturgeschichte zweifach verewigt: erstens durch die an ihn gerichtete Widmung seines Vaters zu dessen Poetik und zweitens dadurch, daß er den Kommentar seines Vaters zu Theophrast, den Robert ConSTANTIN bearbeitet hatte, herausgab.

Somit kommen wir zu Robert Constantin, den Gessner gleichfalls in seinem Brief an SKaLIC erwähnt, und bei ihm wollen wir uns etwas länger aufhalten.

Nach Jöcher ${ }^{39}$ wurde Robert Constantin um 1530 in Caen geboren, wo er 1564 Doktor der Medizin wurde. Nachher durchreiste er ganz Europa, ließ sich dann in Deutschland nieder, von wo er nach einiger Zeit in seine Vaterstadt als Professor Humaniorum berufen wurde. Wegen seines Übertritts zum Protestantismus und seiner im protestantischen Geist gehaltenen Vorlesungen über die Episteln Pauli mußte er neuerlich nach Deutschland auswandern, wo er am 27. Dezember 1605 starb. Thuanus meldet, daß er 103, andere, daß er 110 Jahre gelebt habe. Er war in der hebräischen, griechischen und lateinischen Sprache, wie auch in der Botanik und Medizin

${ }^{36}$ Vgl. Fosses, Crato von Krafftheim (in Studien zur Geschichte der Medizin, Stuttgart 1909, S. 29).

${ }^{37}$ Gessner war übrigens nicht der einzige, der an diese verwandtschaftliche Beziehung glaubte. In seinen beiden Briefen an SKaLIC, vom 20. August 1560 und 1.September 1561, spricht CäLIus Secundus Curio gleichfalls seine Bewunderung für SKalic' «Verwandten» Julius Caesar Scaliger aus (vgl. Satyrae philosophicae, S. 632 und 634). Und dasselbe sehen wir in einem Briefe Schegks (ohne Datum), in dem er von Jul. Caes. Scaliger sagt: «Tuae stirpis et prosapiae» (ibid. S. 639).

${ }^{38}$ Vgl. Joseph Justus Scaliger, Epistolae, Lugd. Batav. 1627, S. 49.

${ }^{39}$ Op. cit., Bd. I, S. 2069. 
sehr erfahren. Er verfaßte zahlreiche griechische und lateinische lexikographische Werke sowie einige medizinische Werke: In Corn. Celsum de re medica, In Quinti Sereni Sammoniaci poema medicinale und In Rhemnii Fannii poema de ponderibus et mensuris ${ }^{40}$. Auch versah er den Kommentar des Amatus Lusitanus zu Dioscorides mit wertvollen Anmerkungen. Er war ein Schüler Julius Caesar Scaligers, in dessen Haus er eine Zeitlang lebte und dessen literarischen Nachlaß er nach dessen Tode herausgab. So besorgte er die Herausgabe von Scaligers Poetik ${ }^{41}$, die ja gerade zur Zeit der Abfassung von Gessners Brief in Genf erschienen war, wie dies GessNER ausdrücklich in seinem Brief erwähnt. Sodann gab er Scaligers Kommentare zu Theophrast heraus ${ }^{42}$, allerdings nicht so bald, wie Gessner dies Skalic in Aussicht stellte, sondern erst einige Jahre später.

Beziehungen zwischen Constantin und Gessner bestanden schon lange vor Abfassung dieses Briefes. Bereits 1555 gab Constantin einen Index zu Gessners großem bibliographischen Werk heraus ${ }^{43}$. Irgendwelche Beziehungen zwischen Constantin und SKalic sind nicht bekannt.

Merkwürdig erscheint immerhin, daß Gessner 1561 Constantin als Jüngling bezeichnet. Das Wort «juvenis» wird hier wohl «junger Mann» zu bedeuten haben, was angesichts der Tatsache, daß Constantin damals im 31.Jahre stand, gleichfalls unangebracht ist. War doch Constantin um vier Jahre älter als Skalic, und doch fällt es Gessner nicht ein, diesen als «juvenis» zu behandeln. Sollte die Angabe stimmen, daß Constantiv nicht 1530, sondern 1502 geboren ist, und diese Angabe finden wir in zahlreichen Quellen, dann war ja Constantin 1561 noch viel weniger ein junger Mann oder gar ein Jüngling, sondern ein Mann von 59 Jahren!

Nun schreibt Gessner in seinem Brief an Skalic, Julius Caesar ScaLIGER hätte Constantin einige seiner Schriften hinterlassen, die dieser demnächst herausgeben werde und die er, wie wir bereits erwähnten, auch tatsächlich herausgab. Es steht fest, daß Julius Caesar Scaligers Sohn JosePh Justus, der nachmalige berühmte Philologe, der seinen Vater weit

${ }^{40}$ Vgl. die Bibliographie seiner Werke bei Jöcher (l. c.), MANGet (op. cit., I/2, S. 114) und SPACHIUs (op. cit., passim).

${ }^{41}$ Poetices libri septem ad Sylvium filium, Genevae, apud J. Crispinum, 1561.

42 Commentarii et animadversiones in sex libros de causis plantarum Theophrasti, Genevae, apud J. Crispinum 1566, und Animadversiones in historias Theophrasti cum Roberti Constantini ad easdem annotationibus, Lugduni, apud Johan. JAC. Junt AM F., 1584.

${ }^{43}$ Nomenclator insignium scriptorum, quorum libri extant vel manuscripti, vel impressi, Indexque totius Bibliothecae atque Pandectarum Gesneri, Paris apud ANDr. WeCheLum, 1555. 
übertraf, bereits seit 1555 der ständige Mitarbeiter und Gehilfe seines Vaters war. Nichts wäre logischer gewesen, als daß der Vater den Sohn mit der Herausgabe seines literarischen Nachlasses betraut hätte. Statt dessen tritt Robert Constantin das Erbe an! Was mag den alten Scaliger bewogen haben, seinen eigenen, höchst talentierten Sohn auf solche Weise auszuspielen? War es bloß der Umstand, daß Constantin um zehn Jahre älter und demnach erfahrener war? Joseph Justus mußte dies als kränkende Zurücksetzung empfinden und das mag vielleicht die Ursache sein, daß er auf RoBert Constantin nicht gut zu sprechen war. Einmal erklärt er ihn als ausgemachten Narren ${ }^{44}$ und ein anderes Mal äußert er ganz unumwunden, daß sein Dictionarium nichts wert sei ${ }^{45}$. GESSNER dagegen hält viel von Constantins Gelehrsamkeit und lobt auch seine Bemühungen um die Herausgabe von Scaligers nachgelassenen Schriften.

Kehren wir nun zu Gessners Brief zurück! Die nun folgende Ermahnung Gessners an SKalic, die auf einem separaten Blatt geschrieben war, wird sich wohl auf die Gerüchte von SKaLIG' Fälschertätigkeit beziehen. Als ob Gessner geahnt hätte, Skalic werde seinen Brief veröffentlichen, schreibt er diese sicherlich nicht für die Öffentlichkeit bestimmten Dinge auf einen eigenen Zettel. Oder war es SKalic, der diese delikaten Stellen bei der Wiedergabe von Gessners Brief wegließ? In diesem Falle hätte er ja auch die Andeutung dieser peinlichen Nachricht ganz weglassen können. Um jene Zeit war das Gerücht von SKalic' Fälschungen schon ziemlich verbreitet, und es wird sich wohl um diese Angelegenheiten handeln, wenn Gessner schreibt, er hätte aus irgendeinem Briefe Unangenehmes über SKaLIC erfahren.

Diesen Brief sandte Gessner durch einen Polen, Sebastian Pecuinus, der weiter nicht bekannt ist. Auffallend ist es, daß Gessner in der Unterschrift neben seinem Namen sein Pseudonym «Philiatros» setzte. Soweit bekannt, hat er sich niemals in seinen Briefen so unterzeichnet. Nicht von der Hand zu weisen ist die Annahme, daß sich Gessner auf diese Weise als der Verfasser des Werkes Thesaurus Evonymi Philiatri de remediis secretis zu erkennen geben wollte ${ }^{46}$. Wer Skalic' Neigungen kennt, wird es für wahrscheinlich halten, daß er dieses Werk gekannt hat.

44 «R. Constantin qui a fait un lexicon n'a pas plus de dix ans plus que moy; c'est un grand fou» (Scaliger, Scaligerana, S. 101).

45 «Constantini Dictionarium non valet» (ibid.).

${ }^{46}$ An diese Möglichkeit glaubt Herr Dozent Dr. Bernhard Mirt in Zürich, wie er mir brieflich mitteilt, ohne in dieser Annahme mehr als eine Vermutung zu sehen. 
Von den beiden in der Nachschrift genannten Männern, denen Gessner seine Grüße sendet, ist Schegk der bekanntere. Jakob Schegk (SchegcIUs, eigentlich Degen), geboren 1511 in Schorndorf (Württemberg), be-. gann als Magister artium 1531 in Tübingen öffentliche Vorlesungen über lateinische Sprache und Literatur zu halten, widmete sich dann der Theologie und studierte schließlich Medizin. 1539 wurde er Doktor der Medizin und bald darauf Professor der Medizin in Tübingen, wo er dreißig Jahre lang wirkte. Er war ein Anhänger des Aristoteles, schrieb auch über Geschichte, Mathematik, scholastische Philosophie und Medizin und hinterließ einige medizinische Schriften. Er starb $1587^{47}$. Daß zwischen Skalic und SchegK Beziehungen bestanden, beweist ein Brief des letzteren (ohne Datum), den wir bereits erwähnt haben und in dem Schegk seiner Bewunderung für Julius Caesar Scaliger Ausdruck gibt ${ }^{48}$. Diese Bewunderung Schegks für Scaliger muß Gessner bekannt gewesen sein, sonst hätte er ihn ja nicht durch Skalic über das baldige Erscheinen einiger Werke ScaLIGERS benachrichtigt.

Die zweite Persönlichkeit, der Gessner durch Skalic Grüße sendet, GABLER, ist weniger bekannt. SKalic erwähnt ihn übrigens an einer anderen Stelle mit Nennung seines Vornamens Venerandus ${ }^{49}$. In den Matrikeln der Tübinger Universität befindet sich eine Eintragung, derzufolge Venerand Gabler aus Nürtingen in Württemberg (Venerandus Gablerus Nurtingensis) am 2. November 1536 immatrikuliert wurde ${ }^{50}$. Im Jahre 1544 wurde er Doktor der Medizin ${ }^{51}$. Näheres über ihn, besonders über seine Beziehungen zu GESSNER, ist nicht bekannt.

Zum Schluß einige Worte über die in Gessners Nachschrift erwähnten Werke Scaligers, deren baldige Herausgabe Gessner zu fördern verspricht. Von seiner Poetik war bereits die Rede. Welches Werk unter dem Titel Nobiles exercitationes gemeint ist, ist nicht klar. Sollte damit eine Neuauflage seiner gegen Cardanus' Werk De subtilitate gerichtete Schrift Exercitationes gemeint sein? Die Kommentare zu den Schriften des Theophrast haben wir gleichfalls erwähnt - sie erschienen allerdings erst einige Jahre später. Ebenso vergingen noch einige Jahre, bevor Scaligers Kommen-

${ }^{47}$ Biographisches Lexikon der hervorragenden Ärzte, Bd. V, S. 59.

${ }^{48}$ Satyrae philosophicae, S. 639.

${ }^{49}$ ibid. S. 153.

${ }^{50}$ Urkunden zur Geschichte der Universität Tübingen aus den Jahren 1476 bis 1550, Tübingen 1877, S. 664 .

51 ibid. 
tare zu Aristoteles erschienen. Der erwähnte Thesaurus linguae Graecae des Robert Constantin wird wohl dessen Lexicon graeco-latinum (Genevae 1562) sein.

Es ist jedoch nicht nur der Inhalt dieses Briefes Gessners an SKalic, der für uns von Interesse ist, sondern auch die alleinige Tatsache, daß zwischen beiden eine Beziehung bestand, verdient eine kurze Besprechung und eine Untersuchung des Ursprungs dieser Beziehung.

Nach Notizen Gessners besuchte Skalic Gessner am 21. April 1559 in Zürich in Begleitung eines protestantischen Theologen, dessen Name nicht bekannt ist. Dagegen entnehmen wir einem Briefe Skalic an Heinrich Bullinger vom 9. Mai 1558, daß SKalic soeben von einem Besuch in Zürich nach Tübingen zurückgekehrt ist, woraus wir schließen können, daß es sich in der erwähnten Notiz um einen Irrtum handelt und daß SKALIG tatsächlich am 21. April 1558 bei GEssner war. In einem zweiten Brief an Bullinger vom 11. März 1560 finden wir einen Vermerk von SKalic' Hand: «Salve meo nomine Gesnerum.» Das sind die einzigen bisher bekannten Hinweise auf die bestehenden Beziehungen Gessners zu SKalic ${ }^{52}$. Außer unserem Brief sind keinerlei Briefe Gessners an Skalic oder Skalic' an GESSNER gefunden worden.

Immerhin darf noch eine zweite Zusammenkunft Gessners mit Skalic, und zwar im Jahre 1559, angenommen werden. In diesem Jahr war GessNER in Augsburg, wo gerade der große Reichstag abgehalten wurde, um dem Kaiser Ferdinand I., dem er das vierte Buch seines zoologischen Werkes gewidmet hatte, auf dessen eigenen Wunsch vorgestellt zu werden. Die Rückreise führte über Tübingen und Straßburg, wo alte Bekanntschaften erneuert und neue angeknüpft wurden ${ }^{53}$. Es ist sehr wahrscheinlich, daß Gessner bei dieser Gelegenheit auch Skalic in Tübingen aufsuchte und dessen Besuch vom vorigen Jahr erwiderte.

Skalic' Beziehung zu Gessner war nicht seine einzige Beziehung zur Schweiz. Unter den Briefen, die SKaLIC in seinen Büchern veröffentlicht hat, befinden sich sieben Briefe von Schweizern. Ihre Absender sind: Petrus Martyr (Zürich, 20. Mai 1558 und 18. August 1560) ${ }^{54}$, Heinrich

${ }^{52}$ Die Kenntnis von diesen in der Zürcher Zentralbibliothek befindlichen Notizen verdanke ich Herrn Dozenten Dr. Bernhard Mrte in Zürich, der mir auch einige weitere sehr wertvolle Angaben brieflich mitteilte, wofür ihm an dieser Stelle mein verbindlichster Dank ausgedrückt sei.

${ }^{53}$ MäHLY in der Allgemeinen Deutschen Biographie, Bd. IX, S. 107.

${ }^{54}$ Satyrae philosophicae, S. 598 und 601. 
Bullinger (Zürich, 13. Mai 1558) ${ }^{55}$, Theodor Bibliander (Zürich, 13. Mai 1558 und 17. August 1560) ${ }^{56}$ und Caelius Secundus Curio (Basel, 20. August 1560 und 2. September 1561) ${ }^{57}$. Der Umstand, daß drei von diesen Briefen im Mai 1558 aus Zürich abgesandt wurden, mag mit dem oben erwähnten, im April 1558 stattgefundenen Besuch SKaLIG' in Zürich zusammenhängen. In allen diesen Briefen handelt es sich um rein theologische Fragen, und es wäre der Mühe wert, wenn von berufener Seite die Beziehungen SKalic' zu den Schweizer Reformatoren eingehend untersucht würden. Besonders eng verbunden waren die südslawischen Protestanten mit Bullinger, dessen Schriften von ihren Führern eifrigst studiert wurden. SKalic selbst war ein großer Verehrer Bullingers, wie wir dies auch einem Brief entnehmen, den SKaLIC am 11. März 1560 an ihn richtete, und in dem es u.a.heißt: «Tu BULLINGERE praestitisti multa, cuius scripta magnifacio semper.» In diesem Brief spricht SKalic auch seine Verehrung für zahlreiche andere Schweizer Theologen aus, so für Petrus Martyr, Bibliander, Gwalter, Ogchino, Viret, Curio, Borrhaus, Sulzer usw. ${ }^{53}$. Die Tatsache, daß zwischen Bullinger und Gessner die engsten Beziehungen bestanden, legt den Gedanken nahe, daß Bullinger es war, der Gessner und Skalic zusammengebracht hatte.

Wenn wir von SKaLIc' Beziehungen zur Schweiz sprechen, dann dürfen wir auch nicht an seine Beziehung zu Johannes Oporin, dem ehemaligen Paracelsus-Schüler und nachmaligen Basler Buchdrucker, vergessen, der im Jahre 1559 Skalic' Enzyklopädie druckte. In diesem Werk befindet sich ein Dankschreiben SKalic' an OpORIN, in dem er ihn nicht nur als Buchdrucker, sondern auch als Freund und hervorragenden Menschen lobt. Ihm widmet er auch sein Encomium scientiarum mit den Worten: «... quamobrem pro primo meo erga te amoris indicio, hoc Encomium, qui pari encomio dignus es, ut sincere et candide suscipias velim. ${ }^{59}$

Auch die Beziehungen Gessners zu Kroatien beschränken sich nicht bloß auf seine Bekanntschaft mit SKalic. Unter den Männern, zu denen Gessner Beziehungen hatte, finden wir noch einen zweiten Kroaten, und zwar Petrus Paulus Vergerius, den ehemaligen Bischof von Capo

55 ibid. S. 603.

56 ibid. S. 605 und 609.

57 ibid. S. 631 und 633.

${ }^{58}$ Dieser Brief befindet sich in der Zürcher Zentralbibliothek. Eine Abschrift verdanke ich gleichfalls Herrn Doz. Dr. MrLt.

${ }^{59}$ Miscellaneorum tomus II, S. 688. 
d'Istria $^{60}$. Bereits 1551 erwähnt ihn GESSNER in seinem zoologischen Werk unter den Männern, die ihm bei der Abfassung dieses Werkes behilflich waren, indem sie ihm Abbildungen, Beschreibungen oder Benennungen von Tieren einsandten oder mündlich mitteilten ${ }^{61}$. Ihm wird es GESSNER wohl zu verdanken haben, daß er in diesem Werk auch einige kroatische Tiernamen mitzuteilen imstande war ${ }^{62}$. Allerdings faßt er unter dem Begriff «Nomina illyrica» nicht nur kroatische, sondern überhaupt slawische Tiernamen zusammen ${ }^{63}$, doch finden wir unter den angeführten Namen viele, die rein kroatisch sind.

Gessne rs Brief an Skalic gibt uns also Gelegenheit, eine Reihe von neuen Tatsachen kennenzulernen, die für die Geschichte der Wissenschaft im 16. Jahrhundert nicht ohne Bedeutung sind. Wir gehen dabei von der Voraussetzung aus, daß Gessners Brief auch tatsächlich echt und keine Fälschung SKalic' ist. Immerhin müßte man daran denken, daß ein Mensch, der selbst vor Fälschung von öffentlichen Dokumenten nicht zurückschreckte, skrupellos den Brief eines Zeitgenossen fälschen mochte, um dadurch das Ansehen zu beweisen, das er bei einem solchen Gelehrten, wie Gessne r es war, genoß. Diese Bedenken müssen jedoch fallengelassen werden und zwar angesichts folgender Tatsachen:

1. Skalic, dessen Beziehungen zu den bedeutendsten Gelehrten seiner Zeit sehr ausgedehnt waren, hatte es nicht nötig, den Brief eines solchen zu fälschen, da er ja mit jedem einzelnen jederzeit in einen Briefwechsel treten konnte.

2. Die Tatsache, daß Skalic 1558 Gessner (nach dessen eigenen Notizen) einen Besuch abgestattet hatte, beweist ja an und für sich, daß zwischen beiden Beziehungen bestanden haben.

3. SKalic konnte es nicht riskieren, einen gefälschten Brief Gessners zu dessen Lebzeiten in einem seiner Werke zu veröffentlichen, da er ja Gefahr laufen mußte, daß Gessner diese Fälschung entdeckte.

${ }^{60}$ Vergerius wurde 1498 in Capo d'Istria geboren, studierte in Padua Theologie und Philosophie und wurde 1536 Bischof von Capo d'Istria. Seit 1543 war er ein Anhänger der Reformation und mußte deshalb flüchten. Von 1550 bis 1553 war er Pfarrer in Vicosoprano (Graubünden), ging dann nach Zürich und starb 1565 in Tübingen.

${ }^{61}$ Historia animalium, lib. I (De quadrupedibus), Tiguri 1551, S. 13.

${ }^{62}$ Vgl. ibid. S. 28.

${ }^{63}$ Er sagt selbst: «Extenditur autem haec lingua hodie per innumeras gentes. Bohemi, Poloni \& Moscovitae peculiaria quaedam habent nomina in hac lingua, ut plerunque notavimus.» 
4. Wäre Gessners Brief eine Fälschung Skalic', dann hätte dieser wahrscheinlich jenen Passus nicht geschrieben, in welchem von gewissen Beschuldigungen gegen SKaLIC die Rede ist, sondern der Brief wäre vielmehr ein Panegyrikus Skalic' geworden.

Wir haben also keinen Grund, an der Echtheit dieses Briefes zu zweifeln, und er besitzt daher vollen dokumentarischen Wert.

$\mathrm{Zu}$ einer neugefundenen paracelsischen Handschrift von Toxites (Michael Schütz) anno 1577:

Von den offenen Schäden vnnd Geschweren

Von J. StrebeL, Luzern

Prof. Karl Sudhoff berichtet im 4.Band der Sämtlichen Werke des Paracelsus in der Einleitung (S. XXII, 1931) über einen «allerneusten Fund» zu den erstmalig in deutscher Sprache im Sommersemester 1527 in Basel gehaltenen Vorlesungen über die chirurgischen Erkrankungen: Von Apostemen, Geschwären, offenen Schäden, Sironen und andern Gewächsen, und verweist auf die diesbezügliche Fußnote S. 657, wo folgendes vermerkt ist: "Während der Korrektur des textkritischen Anhangs (zum 4. Bd.) erfahre ich von Herrn Kollege Mitt in Zürich, daß in Conr. Gessners Handschrift Thesaurus medicinae practicae auf der dortigen Zentralbibliothek, von ihm selbst oder Kaspar Wolf hergestellte Auszüge aus 18 Kapiteln dieser chirurgischen Vorlesung sich finden, die nach einer andern Vorlesungsnachschrift gearbeitet scheinen. Ergeben sich bei ihrer Nachprüfung wichtige Besserungen, so sollen sie dem 14. Band nachgetragen werden»; was nicht der Fall ist, da der 14. Band nichts mehr davon erwähnt. Hier sei kurz über einen «allerneusten Fund» aus der Bürgerbibliothek Luzern berichtet, der, bis anhin völlig unbekannt, aus vier Gründen eine höhere Dignität als der zürcherische beanspruchen darf: er stammt aus der Hand des Humanistenarztes Michael Schürz, genannt Toxites, selbst; er umfaßt die ganze Vorlesung der Capita LXIII, nicht nur 18 Kapitel; er ist, im Gegensatz zu den teilweise in gräßlichem Schülerlatein redigierten zwei Hörernachschriften in deutscher Sprache, auch in den Rezepten, abgefaßt; ferner dürfen wir in diesem Manuskript der Bürgerbibliothek Luzern die Origi- 